Revista Colombiana de Obstetricia y Ginecología Vol. 62 No. 2 • Abril-Junio 2011 • (196-200)

Reporte de caso

\title{
HEMORRAGIA FETOMATERNA MASIVA CON PATRÓN CARDIOTOCOGRÁFICO SINUSOIDAL. PRESENTACIÓN DE UN CASO Y REVISIÓN DE LA LITERATURA
}

\author{
Massive fetomaternal hemorrhage with \\ cardiotocographic sinusoidal rhythm. A case \\ presentation and literature review \\ Daniel Abehsera-Davó, M.D.*, Carlos Iglesias-Sánchez, M.D.**, \\ Fernando Magdaleno Dans, M.D.***, Antonio González-González, M.D.**** \\ Recibido: noviembre 11/10 - Aceptado: mayo 3/11
}

\section{RESUMEN}

Introducción: la transfusión fetomaterna masiva (TFM) es una entidad con una elevada morbilidad y mortalidad fetal, suele cursar con una disminución en la percepción de los movimientos fetales por parte de la madre, y la presencia de un patrón cardiotocográfico sinusoidal fetal, asociado a la anemia fetal. No obstante, ambas situaciones tienen muy baja especificidad. Se presenta un caso clínico con el objetivo de revisar la exactitud del patrón sinusoidal en el diagnóstico de anemia fetal.

Materiales y metodos: se presenta el caso de una gestante de 36 semanas que fue atendida en el Hospital Universitario La Paz (complejo hospitalario de tercer nivel que forma parte del conjunto de hospitales públicos en España), en la que tras presentarse un patrón cardiotocográfico no tranquilizador, se realizó una inducción del

* Médico interno residente. Hospital Universitario La Paz. Madrid (España).Correo electrónico: danielabehsera@hotmail.com

** Médico interno residente. Hospital Universitario La Paz. Madrid (España).

*** Jefe de Sección de Obstetricia. Hospital Universitario La Paz. Madrid (España).

***** Jefe de Servicio de Obstetricia. Hospital Universitario La Paz. Madrid (España). parto en el que las pruebas habituales de bienestar fetal resultaron insuficientes para el diagnóstico de sufrimiento fetal. Tras un parto eutócico se comprobó la presencia de una anemia neonatal grave, comprobándose la presencia de un gran volumen de sangre fetal en la sangre materna mediante el test de Kleihauer Betke. Se hace una revisión de los artículos publicados en los últimos 10 años en las base de datos Medline vía PubMed, en español e inglés.

Conclusión: la monitorización fetal intraparto podría ser útil en el diagnóstico de la hemorragia fetomaterna masiva, aunque se deben hacer estudios más amplios para determinar la exactitud diagnóstica.

Palabras clave: transfusión fetomaterna, frecuencia cardíaca fetal, sangre fetal, hipoxia fetal.

\section{SUMMARY}

Introduction: massive fetomaternal transfusion (MFT) is an entity having high fetal morbidity and mortality; it usually involves the mother's reduced perception of fetal movements and the presence of a cardiotocographic fetal sinusoidal rhythm, associated with fetal anemia. However, both 
situations have very low specificity. A clinical case is presented here to arouse interest in reviewing the precision of the sinusoidal rhythm when diagnosing fetal anemia.

Materials and methods: the case of a 36-weeks pregnant mother is presented; she attended $\mathrm{La} \mathrm{Paz}$ teaching hospital (a third-level hospital forming part of the Spanish public hospital system). After presenting a non-reassuring cardiotocographic heart rate pattern, birth was induced in which the usual fetal wellbeing tests proved insufficient for diagnosing fetal suffering. The presence of serious neonatal anemia was shown following a eutocic delivery, the Kleihauer-Betke test proving the presence of a large volume of fetal blood in the mother's blood. Articles published in both Spanish and English during the last 10 years in the Medline database were reviewed via PubMed.

Conclusion: intradelivery fetal monitoring could be useful in diagnosing massive fetal-maternal hemorrhage, even though broader studies should be carried out for determining diagnostic precision. Key words: fetomaternal transfusion, fetal heart rate, fetal blood, fetal hypoxia.

\section{INTRODUCCIÓN}

La transfusión fetomaterna se define como el paso de eritrocitos fetales a la circulación materna. Aunque se ha informado que acontece hasta en el 40-50\% de las gestaciones, en el 98\% de los casos la cantidad de la hemorragia es inferior a $0,1 \mathrm{ml}$, lo cual no influye en el resultado perinatal. ${ }^{1}$ La transfusión fetomaterna masiva (TFM) es la pérdida de más de 80-150 ml, aproximadamente el 50\% de la volemia fetal, y tiene una incidencia aproximada de 1 de cada $1000-2800$ gestaciones. ${ }^{1-3}$ La TFM va aumentando a medida que avanza la gestación, teniendo su máxima expresión durante el parto. ${ }^{4}$ En la mayoría de los casos, acontece en gestaciones cercanas al término y sin factores de riesgo, ${ }^{5}$ y suele cursar con una clínica inapreciable. ${ }^{1}$ Cuando el volumen transfundido alcanza o sobrepasa los $20 \mathrm{ml} / \mathrm{Kg}$, la TFM causa anemia fetal y acarrea severas consecuencias para el feto o el recién nacido. ${ }^{6}$
Las graves consecuencias derivadas de una TFM convierten a esta entidad en una patología de gran importancia a pesar de su baja incidencia. La causa concreta de una gran TFM suele quedar indeterminada en la mayoría de los casos. Las causas conocidas de hemorragia fetomaterna son diversas, y sobre todo responden a procesos invasivos como biopsia corial, cordocentesis y aborto electivo. ${ }^{7}$

Presentamos el caso de una gestante sin factores de riesgo, en la que se produjo una TFM asociada a un patrón cardiotocográfico sinusoidal. Se inició el estudio haciendo una revisión de la literatura pertinente con la utilidad de las pruebas empleadas para el diagnóstico de la entidad.

\section{CASO CLÍNICO}

Mujer de 29 años, primigesta, que fue atendida en el Hospital Universitario La Paz que hace parte del complejo hospitalario público de tercer nivel, ubicado en Madrid (España). Acudió a urgencias en la semana 36 y 2 días de gestación, refiriendo dolor abdominal, de escasa intensidad, localizado en hipogastrio que se irradia a fosa ilíaca izquierda. No presentó dolor a la palpación abdominal. El feto se encontraba en situación longitudinal, presentación cefálica y bolsa íntegra. Se realizó un registro cardiotocográfico externo durante 20 minutos, en el que se observó una frecuencia cardíaca fetal (FCF) basal de 140 lat/ min, ausencia de ascensos, variabilidad disminuida (compatible con patrón cardiotocográfico sinusoidal minor) y una desaceleración de 2 minutos de duración de inicio sincrónico con la contracción, de hasta 75 lat/min y con lenta recuperación posterior, compatible con DIP variable atípico (figura 1). La dinámica uterina fue irregular, con 1-3 contracciones cada diez minutos de 20-50 mm Hg. Su ingreso se decidió en la sala de dilatación por el registro cardiotocográfico patológico y cérvix favorable en la gestación pretérmino, con la intención de finalizar la gestación.

A su llegada a la sala de partos se realizó amniorrexis artificial y una monitorización interna, en donde se encontró líquido amniótico claro. Luego, 
Figura 1. Registro cardiotocográfico realizado en urgencias que motiva el ingreso.

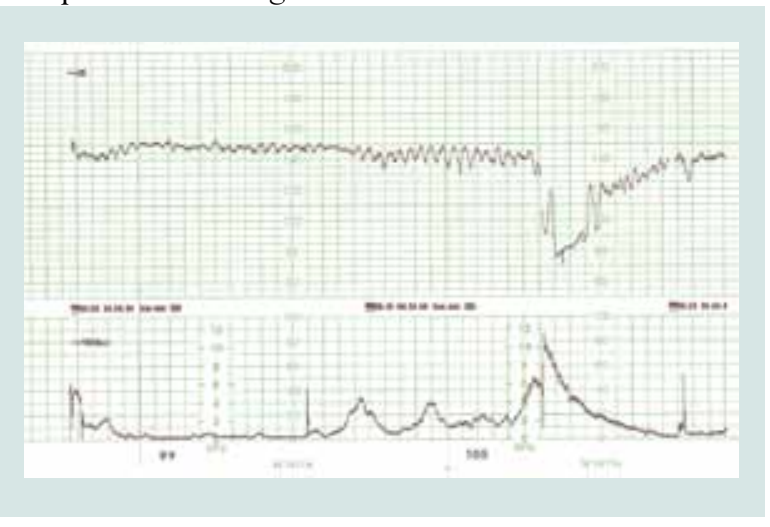

se procedió a la estimulación del parto mediante oxitocina IV y se aplicó analgesia epidural por solicitud de la paciente. Desde la amniorrexis artificial hasta la expulsión fetal transcurrieron seis horas. Una hora después de la llegada de la paciente a la sala de partos, se optó por realizar una microtoma de sangre fetal debido a la aparición de más desaceleraciones variables atípicas, ausencia de ascensos, y persistencia de un ritmo silente, con tramos intermitentes de sinusoidal minor (figura 2); se obtuvieron dos muestras de sangre, en las que se halla un resultado de $\mathrm{pH}$ fetal de 7,32 y 7,33. Este patrón cardiotocográfico se perpetuó hasta el expulsivo.

Figura 2. Registro cardiotocográfico intraparto.

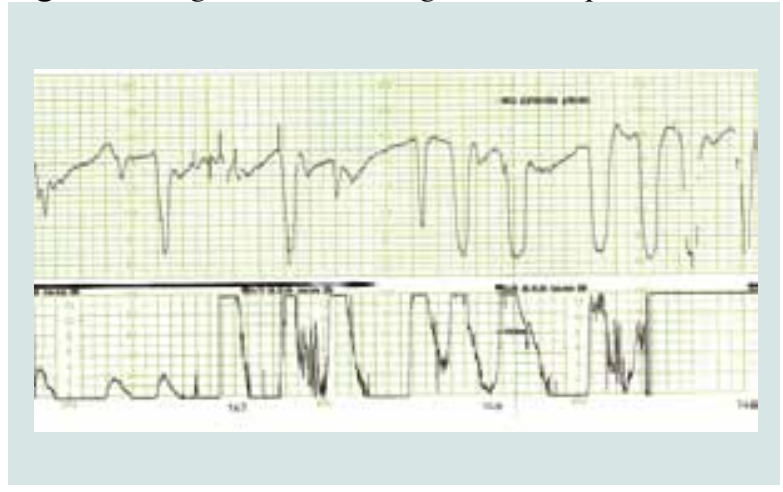

Durante el expulsivo que duró 20 minutos, el trazado de FCF fue similar, lo que se asoció con graves desaceleraciones periódicas y una ausencia de variabilidad a corto plazo (figura 3). El parto fue
Figura 3. Registro cardiotocográfico durante el período de expulsivo.

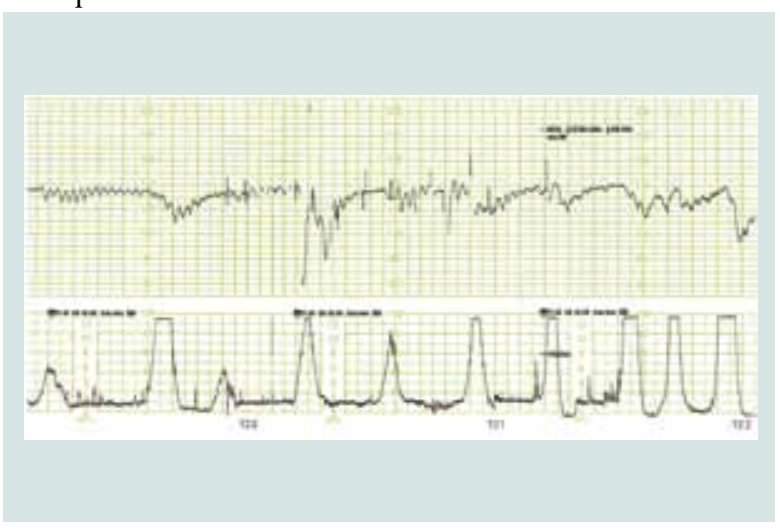

eutócico de una mujer viva, de peso adecuado para edad gestacional $(2500 \mathrm{~g})$. La puntuación del test de Apgar al minuto fue de 3, y a los 5 minutos de 4. El recién nacido presentó una palidez muy intensa e hipoactividad marcada; con los latidos cardíacos normales pero con escaso esfuerzo respiratorio, por lo que requirió de intubación y traslado a la sala de cuidados intensivos. El pH del cordón posparto mostró una gran disparidad entre los valores obtenidos en la arteria umbilical $(6,87$ con acidosis mixta) y en la vena umbilical $(7,20)$. La hemoglobina fetal fue de 3,9 $\mathrm{g} / \mathrm{dl}$ con un hematocrito del 11\%.

Ante la sospecha de TFM, se realizó el test de Kleihauer-Betke, el cual mostró que en la sangre periférica materna había un gran número de hematíes fetales con un volumen estimado de hemorragia de 130-150 ml. Por lo que fue necesario realizar una transfusión de sangre al neonato que posteriormente evolucionó de forma favorable, recibiendo el alta a los 10 días.

\section{MATERIALES Y MÉTODOS}

Se realizó una revisión de los artículos publicados en los últimos 10 años en las base de datos Medline vía PubMed, en español e inglés, y en libros de la especialidad relativos a los términos "massive fetomaternal hemorrhage", "sinusoidal patterns" y "fetal anemia". Se seleccionaron reportes, series de casos y artículos de revisión, y se revisaron más de 200 títulos relacionados, de los cuales fueron revisados a texto completo un total de 12 artículos. 


\section{DISCUSIÓN}

El diagnóstico de la TFM se sospecha clínicamente por una disminución en la percepción de los movimientos fetales por parte de la madre. ${ }^{5}$ El registro cardiotocográfico nos permite sospechar su diagnóstico por medio de la identificación de un patrón sinusoidal, lo que podría sugerir anemia fetal, y un resultado final de TFM. ${ }^{8}$ Este patrón fue descrito por Modanlou y Freeman en 1982 y se caracteriza por: 1) frecuencia cardíaca basal y uniforme, aunque con oscilaciones regulares. Las formas más graves suelen coincidir con una FCF bradicárdica. 2) amplitud de 5 a 15 lat/min (sinusoidal minor), siendo raramente superior (sinusoidal mayor). 3) frecuencia de 2 a 5 ciclos por minuto. 4) variabilidad a corto plazo silente. 5) oscilación de la onda sinusoidal equidistante de la línea de base de la FCF. 6) ausencia de tramos de registro de reactividad o de variabilidad normal de la FCF. ${ }^{2,8}$ En definitiva, el trazado sinusoidal es un patrón especial y extremadamente raro, que se caracteriza por la pérdida de la variabilidad a corto plazo, pero conservándola a largo plazo.

Para que un trazado de FCF pueda ser etiquetado como sinusoidal es necesario que coincida con una variabilidad a corto plazo inferior a 5 lat/min (ritmo silente) y que persista, como mínimo, durante diez minutos; lo que permite descartar muchas pseudosinusoidales.

El peor pronóstico lo tiene el trazado sinusoidal persistente, "mayor" y anteparto, aunque cualquier sinusoidal verdadera tiene mal pronóstico y puede ser premonitoria de muerte fetal próxima. En esta situación, el patrón se correlaciona con una hiperactividad del sistema nervioso autónomo para intentar mantener la homeostasis fetal, aunque antes se pensaba que la causa de este patrón sería la ausencia de control del sistema nervioso autónomo sobre la frecuencia cardíaca, manifestado en fallo cardíaco fetal con disminución del gasto cardíaco o hipoxia tisular. ${ }^{9}$

El patrón sinusoidal real también se puede presentar como una respuesta fetal benigna secundaria a un efecto farmacológico; igualmente, durante los movimientos de succión fetal, puede observarse un patrón pseudosinusoidal. ${ }^{10,11}$ Otras causas de patrón sinusoidal son: preeclampsia, corioamnionitis, anemia materna, así como las de causa idiopática. ${ }^{8}$

Maeda y Nagasawa establecieron en 2004 un sistema automático computarizado para el diagnóstico diferencial entre el patrón cardiotocográfico sinusoidal patológico y fisiológico; llegando en su serie de casos, a una tasa de verdaderos positivos del 100\% y una tasa de falsos positivos del 0\%; no obstante, la serie de casos fue sólo de 16 pacientes de los cuales 9 presentaron un trazado sinusoidal patológico y 7 de ellos uno fisiológico. ${ }^{12}$

En un estudio realizado en el Hospital John Radcliffe (Oxford), se analizaron 72297 registros de 19506 embarazadas, los cuales fueron analizados mediante un algoritmo que permite identificar el patrón cardiotocográfico sinusoidal. Se recogieron 15 registros etiquetados como patrón sinusoidal de 8 de las embarazadas. De estos ocho casos, cinco de ellos fueron casos de anemia fetal; y se distinguían de los otros tres casos por una muy marcada pérdida de la variabilidad a largo plazo en el trazado del registro cardiotocográfico. ${ }^{13}$ De esta manera, se ha podido asociar la pérdida marcada de variabilidad a largo plazo en un patrón sinusoidal con una situación de anemia fetal.

A pesar de que la TFM ha sido relacionada con un patrón cardiotocográfico sinusoidal desde hace más de veinte años, en los últimos años la ecografía Doppler constituye una herramienta de apoyo para el diagnóstico de la anemia fetal, estudiando la velocidad máxima sistólica en la arteria cerebral media. La medición del pico sistólico en la arteria cerebral media presenta una sensibilidad del 100\% y una especificidad del 88\% para el diagnóstico de anemia fetal. ${ }^{14}$

Por otra parte, cabe destacar que la microtoma de sangre fetal sólo diagnostica la depresión neonatal debida a hipoxia fetal, sin ser capaz de 
detectar la pérdida del bienestar fetal que responde a otras causas no hipóxicas, como infección, trauma, prematuridad, fármacos o malformaciones. ${ }^{15}$ En la TFM se podría producir una hemorragia fetal intensa y muy aguda que puede comprometer la salud fetal sin que haya tiempo para que se desarrolle una acidosis. Esto explicaría por qué en el caso presentado, ambas microtomas fetales intraparto se encontraban en el rango de la normalidad. De esta manera, cuando el patrón cardiotocográfico sinusoidal se presente intraparto, la realización de una microtoma de sangre fetal para conocer el valor del pH sanguíneo, sería insuficiente, siendo necesaria la determinación de la hemoglobina y el hematocrito fetales.

\section{CONCLUSIÓN}

El registro cardiotocográfico es un método útil en el diagnóstico de sospecha de TFM, siendo necesaria la utilización de otras herramientas diagnósticas tales como: la medición del pico sistólico en la arteria cerebral media; con el objetivo de finalizar la gestación si fuera necesario.

\section{REFERENCIAS}

1. García JA, Benito V, Reyes D. Transfusión fetomaterna masiva: presentación de 2 casos. Prog Obstet Ginecol 2004;47:191-5.

2. Malcus P, Björklund LJ, Lilja M, Teleman P, Laurini R. Massive feto-maternal hemorrhage: diagnosis by cardiotocography, Doppler ultrasonography and ST waveform analysis of fetal electrocardiography. Fetal Diagn Ther 2006;21:8-12.

3. Dziegiel MH, Nielsen LK, Berkowicz A. Detecting fetomaternal hemorrhage by flow cytometry. Curr Opin Hematol 2006;13:490-5.
4. Choavaratana R, Uer-Areewong S, Makanantakocol S. Fetomaternal transfusion in normal pregnancy and during delivery. J Med Assoc Thail 1997;80:96-100.

5. Carmona E. Hemorragia fetomaterna masiva. Caso clínico. Clin Invest Ginecol Obstet 2005;32:268-71.

6. Rubod C, Deruelle P, Le Goueff F, Tunez V, Fournier M, Subtil D. Long-term prognosis for infants after massive fetomaternal hemorrhage. Obstet Gynecol 2007;110:256-60.

7. Cunningham FG, Gant NF, Leveno KJ, Gilstrap III LC, Hauth JC, Wenstrom KD. Williams Obstetricia. $21^{\text {a }}$. ed. Madrid: Editorial Médica Panamericana; 2003. p. 902-3.

8. Cabaniss ML. Monitorización fetal electrónica. Interpretación. $1^{\mathrm{a}}$ ed. Barcelona: Editorial Masson; 1995.

9. Modanlou HD, Murata Y. Sinusoidal heart rate pattern: Reappraisal of its definition and clinical significance. J Obstet Gynaecol Res 2004;30:169-80.

10. Quijada D, López AJ, Gaitán N, Cerrillos L. Patrón sinusoidal por transfusión fetomaterna: a propósito de un caso. Prog Obstet Ginecol 1999;42:324-7.

11. Valdés E. Rol de la monitorización fetal intraparto en el diagnóstico del sufrimiento fetal agudo. Rev chil obstet ginecol 2003;68:411-19.

12. Maeda K, Nagasawa T. Automatic computerized diagnosis of fetal sinusoidal heart rate. Fetal Diagn Ther 2005;20:328-34.

13. Reddy A, Moulden M, Redman CW. Antepartum high-frequency fetal heart rate sinusoidal rhythm: computerized detection and fetal anemia. Am J Obstet Gynecol 2009;200:40.e1-6.

14. Borrel A, Pereira A, Puerto B. Enfermedad hemolítica perinatal. Prog Obstet Gynecol 2001;44:19-22.

15. Sociedad Española de Ginecología y Obstetricia. Tratado de Ginecología, Obstetricia y Medicina de la Reproducción. 1 ${ }^{a}$. ed. Madrid: Editorial Médica Panamericana; 2003. 